\title{
COMPARATIVE STUDY OF PRIMAL- AND DUAL-MIXED FINITE ELEMENT MODELS FOR CYLINDRICAL SHELLS
}

\author{
EDGÁR BERTótI \\ Institute of Applied Mechanics, University of Miskolc \\ H-3515 Miskolc, Miskolc-Egyetemváros, Hungary \\ edgar . bertoti@uni-miskolc.hu \\ [Received: October 4, 2015, Accepted: November 10, 2015.] \\ Dedicated to Professor Barna Szabó on the occasion of his eightieth birthday \\ and to Professor Imre Kozák on the occasion of his eighty-fifth birthday
}

\begin{abstract}
Analytical and numerical comparisons of a primal-mixed, a dual-mixed and a consistent primal-dual mixed finite element formulation are presented for cylindrical shells using the lowest possible order, constant and linear, polynomial approximations within the framework of the Naghdi shell model. The stiffness matrices and the load vectors of the mixed elements are explicitly derived and compared to each other and to that of the standard displacement-based shell element for axisymmetric deformations. It is pointed out that the stiffness matrices of the dual-mixed and the primal-dual mixed elements can be related to that of the standard shell element through two geometry-, material- and mesh-dependent coefficients. One of these coefficients turns out to be a reliable shear locking indicator which can be used to transform the standard displacement-based shell element into a shear locking-free dual-mixed one, independently of the thickness and the loading of the shell. The numerical comparisons indicate that the dual-mixed element is the only element that gives second-order rates of asymptotic convergence for all the variables, including the bending moment and shear force computations.
\end{abstract}

Mathematical Subject Classification: 05C38, 15A15

Keywords: primal-mixed, dual-mixed, finite element, cylindrical shell, shear locking indicator

\section{INTRODUCTION}

Reliable numerical analysis of structural shells represents one of the biggest challenges in computational mechanics. During the past decades, a large variety of shell formulations and shell finite element models have been developed, investigated and analyzed, both numerically and analytically. Due to the enormously huge number of publications on shell formulations and shell finite elements, it is almost impossible to give a brief overview within a short introduction, even on the most significant contributions. The reader is rather referred to the excellent review of [1] and the textbooks of [2, 3, 4, 5]. Among the current possibilities, the higher-order displacement-based shell 
elements and the low-order mixed shell finite elements seem to be the most reliable ones for thin shells [5, 6, 7.

The appearance of, and the remedies for, the different types of locking phenomenon, including shear and membrane locking, in finite element analyses of beams, plates and shells have been the subject of many research papers as well. Regarding Naghdi-type first-order shear deformation models for shells [8, 9, 10, 11, several shear locking-free elements have been developed and different techniques for avoiding locking problems have also been widely analyzed [12, 13, 4, 5].

Regarding mixed variational formulations and finite element models, the primalmixed elements with continuous displacement approximations and discontinuous surface tractions are more popular than the dual-mixed elements, but they lead to the same low-order rates of convergence for the stresses as the conventional displacementbased models [14. In contrast, the dual-mixed elements require surface traction continuity at the element interfaces and apply discontinuous approximations for the displacements, i.e., a standard stiffness matrix equation with conforming nodal displacements cannot directly be derived from them. However, as the local equilibrium of the surface tractions at the element interfaces is satisfied by the stress approximation of the dual-mixed elements, they result in better rates of asymptotic convergence for the stress variables than those obtained by primal-mixed elements.

The locking-free property of a shell finite element is understood in the sense that no degradation in the rates of convergences of the displacement and stress variables appears when the thickness of the shell approaches zero. The analytical investigation and explanation of the locking-free behavior of different, displacement-based or mixed, shell elements would require explicit comparisons of their closed form stiffness matrices and load vectors. This, however, seems to be rather hopeless for general shells, due to the complexity of their geometry, the related variational formulations and finite element models. In addition, the discontinuous approximation of the displacements in dual-mixed finite element formulations makes their analytical comparisons to primaland primal-mixed elements rather difficult. This is true even for those cases when the surface traction continuity at the element interfaces is enforced by applying the $\lambda$ multiplier technique, a method often called hybridization [15, 16, as the $\lambda$-multipliers usually correspond to non-conforming nodal displacements. Special exceptions to that rule are the one-dimensional problems like beams and, regarding shells, the cylindrical shell with axisymmetric deformations, where the $\lambda$-multipliers turn out to be the nodal displacements. This fact enables direct analytical comparisons of the element stiffness matrices and load vectors of the dual-mixed formulation to those obtained with the displacement-based and the primal-mixed approaches.

The main goal of the present work is to perform analytical and numerical comparisons of different finite element formulations for cylindrical shells in the framework of the dimensionally reduced Naghdi shell model. The formulations investigated in this paper have much similarity and analogy to those presented for the Timoshenko beam in [17. The strong and the (Galerkin-type) weak formulations of the governing equations for axisymmetric deformations are summarized in Section 2 Among the several 
possibilities, a primal-mixed, a dual-mixed and a consistent primal-dual mixed formulation are investigated in detail, deriving the corresponding stiffness matrices and load vectors analytically, using the lowest possible order of polynomial approximations the formulations permit.

The derivation and comparison of the stiffness matrices and the load vectors of the different finite element models have been performed using the computer algebra system Maple(TM) and the results are summarized in Section 3. The relationships between the element stiffness matrices suggest the introduction of two geometry-, material- and mesh-dependent coefficients denoted by $C_{\mathrm{m}}$ and $C_{\mathrm{s}}$ that can easily be computed for each element in the mesh. To show and compare the approximation capabilities of the formulations and elements considered, Section 4 presents a demonstrative numerical example, a clamped-clamped shell subjected to uniform load. In the concluding remarks a recipe is given for how the stiffness matrices of the standard and primal-mixed formulations can be transformed, applying the shear locking indicator $C_{\mathrm{s}}$, to obtain shear locking-free finite element solutions with second-order rates of asymptotic convergences in all the variables, i.e., in the displacements, bending moments and shear forces, independently of the thickness of the shell.

\section{Strong AND WEAK FORMULATIONS FOR THE CYLINDRICAL SHELL MODEL}

A homogeneous circular cylindrical shell of length $L$ and uniform thickness $d$ is investigated in a Cartesian $x y z$ coordinate system. The axis $x$ coincides with the axis of the shell, the middle surface of which is a cylinder of radius $R$ (see Figure 1). The orthonormal basis vectors of the local cylindrical coordinate system, attached to the middle surface, are denoted by $\boldsymbol{e}_{i}, i=1,2,3$, the cylindrical coordinates of an arbitrary point $P$ on the middle surface are $x_{1} \equiv x, x_{2}$ and $x_{3}$, each of them has a dimension of length. The middle surface of the shell is given by $S_{0}:=\left\{\boldsymbol{x} \mid x \in(0, L), x_{2} \in[0,2 R \pi), x_{3}=0\right\}$. We assume that the shell is loaded axisymmetrically, i.e., all the variables used for describing the shell problem are independent of the coordinate $x_{2}$. The torsion problem of the shell is not investigated.

2.1. Strong forms of the governing equations. Applying the main kinematical assumptions of the Naghdi shell model 8, 5, the displacement field of the 3D shell is approximated through the thickness as

$$
\begin{aligned}
& u_{1}\left(x, x_{3}\right)=u(x)+\phi(x) x_{3}, \\
& u_{3}\left(x, x_{3}\right)=w(x),
\end{aligned}
$$

where $u(x)$ and $w(x)$ are the displacements of the middle surface in axial and transverse directions, respectively, and $\phi(x) \equiv \phi_{2}(x)$ is the (small) rotation of the normal to the middle surface at $x \equiv x_{1}$ around the local base vector $\boldsymbol{e}_{2}$. The displacement component $u_{2}$ is identically zero. The non-zero strain components of the shell are given by

$$
e_{11}\left(x, x_{3}\right)=\varepsilon_{11}(x)+\kappa_{11}(x) x_{3},
$$




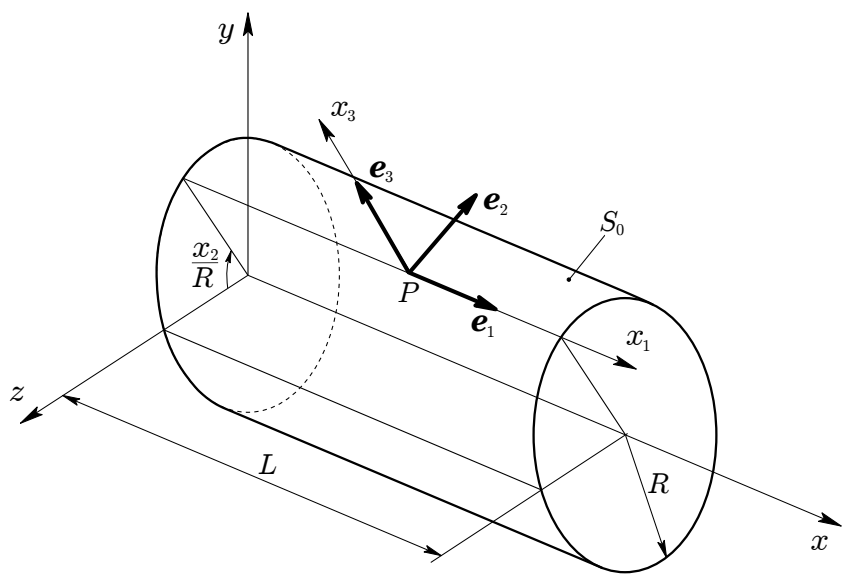

Figure 1. Middle surface of the cylindrical shell

$$
\begin{aligned}
2 e_{13}\left(x, x_{3}\right) & =\gamma_{13}(x), \\
e_{22}\left(x, x_{3}\right) & =\varepsilon_{22}(x),
\end{aligned}
$$

where

$$
\begin{aligned}
\varepsilon_{11}(x) & =u,,_{x}, \\
\gamma_{13}(x) & =w,{ }_{x}+\phi, \\
\kappa_{11}(x) & =\phi,,_{x}, \\
\varepsilon_{22}(x) & =w / R
\end{aligned}
$$

are, respectively, the axial membrane strain, the transverse shear strain, the curvature of the middle surface in the $x_{1} x_{3}$ plane and the circumferential membrane strain (a comma followed by the index $x$ in the subscript denotes differentiation with respect to $\left.x \equiv x_{1}\right)$. Relations $(2.6)-(2.9)$ are the kinematic equations of the dimensionally reduced shell model with Reissner-Mindlin-type kinematics. Note that 2.9$)$ is not a differential equation.

Assuming linearly elastic and isotropic materials and identically zero transverse normal stresses, i.e., $\sigma_{33}=0$, the constitutive relations for the $3 \mathrm{D}$ shell are given by

$$
\begin{aligned}
& \sigma_{11}\left(x, x_{3}\right)=E_{1}\left(\varepsilon_{11}+\nu \varepsilon_{22}\right)+E_{1} \kappa_{11} x_{3}, \\
& \sigma_{22}\left(x, x_{3}\right)=E_{1}\left(\varepsilon_{22}+\nu \varepsilon_{11}\right)+\nu E_{1} \kappa_{11} x_{3}, \\
& \sigma_{13}\left(x, x_{3}\right)=k_{\mathrm{s}} 2 G \varepsilon_{13}=k_{\mathrm{s}} G \gamma_{13}(x),
\end{aligned}
$$

where $E_{1}=E /\left(1-\nu^{2}\right)$ with elasticity modulus $E$ and Poisson ratio $\nu, G$ is the shear modulus and $k_{\mathrm{s}}$ is the shear correction factor. Introducing the stress resultants and couples as

$$
\left\{\begin{array}{l}
N_{11}(x) \\
N_{22}(x) \\
N_{13}(x)
\end{array}\right\}=\int_{-\frac{d}{2}}^{+\frac{d}{2}}\left\{\begin{array}{l}
\sigma_{11} \\
\sigma_{22} \\
\sigma_{13}
\end{array}\right\} \mathrm{d} x_{3}, \quad\left\{\begin{array}{l}
M_{11}(x) \\
M_{22}(x)
\end{array}\right\}=\int_{-\frac{d}{2}}^{+\frac{d}{2}} x_{3}\left\{\begin{array}{l}
\sigma_{11} \\
\sigma_{22}
\end{array}\right\} \mathrm{d} x_{3},
$$


the stress-strain relations of the shell model can be given in the form

$$
\begin{aligned}
& N_{11}(x)=E_{1} d\left(\varepsilon_{11}+\nu \varepsilon_{22}\right), \\
& N_{22}(x)=E_{1} d\left(\varepsilon_{22}+\nu \varepsilon_{11}\right), \\
& N_{13}(x)=k_{\mathrm{s}} G d \gamma_{13}, \\
& M_{11}(x)=E_{1} I_{1} \kappa_{11}, \\
& M_{22}(x)=\nu E_{1} I_{1} \kappa_{11},
\end{aligned}
$$

where $I_{1}=d^{3} / 12$. Due to the assumptions employed, the geometrical and material parameters $I_{1}, E, G$ and $\nu$, as well as $k_{\mathrm{s}}$ are all constants. Note that the above constitutive equations are uniquely invertible and the membrane strains, for example, can be computed from the membrane forces according to the inverse stress-strain relations

$$
\begin{aligned}
\varepsilon_{11}(x) & =\frac{1}{E d}\left(N_{11}-\nu N_{22}\right), \\
\varepsilon_{22}(x) & =\frac{1}{E d}\left(N_{22}-\nu N_{11}\right) .
\end{aligned}
$$

The equilibrium equations of the shell model are

$$
\begin{aligned}
N_{11, x}+p_{1} & =0, \\
N_{13, x}-\frac{1}{R} N_{22}+p_{3} & =0, \\
M_{11, x}-N_{13}+m_{2} & =0,
\end{aligned}
$$

where $p_{1}(x), p_{3}(x)$ and $m_{2}(x)$ are distributed (known) external loads. For thin shells, the distributed moment load $m_{2}(x)$ is usually neglected, as we do so in this paper.

Displacement and stress boundary conditions can be prescribed for the variables $u, w, \phi$ and $N_{11}, N_{13}, M_{11}$, respectively, at both ends of the shell, $x=0$ and $x=L$, by paying attention to the fact that $\left\{u, N_{11}\right\},\left\{w, N_{13}\right\}$ and $\left\{\phi, M_{11}\right\}$ are work-conjugate variables.

2.2. Weak forms of the governing equations. In the subsequent analysis, the constitutive equations (2.14)-2.18) will be satisfied in a strong sense. In addition, to simplify the finite element formulation and to obtain comparable $4 \times 4$ stiffness matrices, the kinematic equation (2.6) and the equilibrium equation (2.21) will also be satisfied in a strong sense. This means that the solution for the axial membrane force $N_{11}(x)$ is obtained by direct integration from $(2.21)$ as

$$
N_{11}(x)=N_{11}(0)-\int_{\xi=0}^{x} p_{1}(\xi) \mathrm{d} \xi
$$

and the axial displacement $u(x)$ will be obtained from 2.6 by integration as well:

$$
u(x)=u(0)+\int_{\xi=0}^{x} \varepsilon_{11}(\xi) \mathrm{d} \xi .
$$


The computation of the strain component $\varepsilon_{11}(x)$ for 2.25$)$ is based on 2.19 , which requires a solution for $N_{22}(x)$. The method of computing the membrane force $N_{22}(x)$ depends on the weak formulation applied, as discussed in Section 3 .

2.2.1. Kinematic equations. The first weak forms of the kinematic equations (2.7)2.8) of the shell model are

$$
\begin{aligned}
\int_{0}^{L}\left(\gamma_{13}-w,_{x}-\phi\right) \delta N_{13} \mathrm{~d} x & =0 \\
\int_{0}^{L}\left(\kappa_{11}-\phi,_{x}\right) \delta M_{11} \mathrm{~d} x & =0,
\end{aligned}
$$

where $\delta N_{13}(x)$ and $\delta M_{11}(x)$ are arbitrary weighting functions (virtual shear force and bending moment). The second weak forms of the kinematic equations are obtained from 2.26 -2.27) by applying the divergence theorem:

$$
\begin{aligned}
\int_{0}^{L}\left(\gamma_{13} \delta N_{13}+w \delta N_{13, x}-\phi \delta N_{13}\right) \mathrm{d} x-\left[w \delta N_{13}\right]_{0}^{L} & =0 \\
\int_{0}^{L}\left(\kappa_{11} \delta M_{11}+\phi \delta M_{11, x}\right) \mathrm{d} x-\left[\phi \delta M_{11}\right]_{0}^{L} & =0 .
\end{aligned}
$$

The kinematic equation 2.9 is not a differential equation, it has only one weak form:

$$
\int_{0}^{L}\left(\varepsilon_{22}-\frac{w}{R}\right) \delta N_{22} \mathrm{~d} x=0,
$$

where $\delta N_{22}(x)$ is an arbitrary weighting function (virtual membrane force).

2.2.2. Equilibrium equations. The first weak forms of the equilibrium equations 2.22)2.23 are

$$
\begin{aligned}
\int_{0}^{L}\left(N_{13, x}-\frac{1}{R} N_{22}+p_{3}\right) \delta w \mathrm{~d} x & =0, \\
\int_{0}^{L}\left(M_{11, x}-N_{13}\right) \delta \phi \mathrm{d} x & =0,
\end{aligned}
$$

where $\delta w\left(x_{1}\right)$ and $\delta \phi\left(x_{1}\right)$ are arbitrary functions (virtual displacements and rotation). The second weak forms of the equilibrium equations are obtained from $2.31-2.32$ by applying the divergence theorem:

$$
\begin{aligned}
\int_{0}^{L}\left(-N_{13} \delta w,_{x}-\frac{1}{R} N_{22} \delta w+p_{3} \delta w\right) \mathrm{d} x+\left[N_{13} \delta w\right]_{0}^{L} & =0 \\
\int_{0}^{L}\left(-M_{11} \delta \phi,_{x}-N_{13} \delta \phi\right) \mathrm{d} x+\left[M_{11} \delta \phi\right]_{0}^{L} & =0
\end{aligned}
$$


2.3. Variational formulations $-\mathbf{a}$ brief overview of the possibilities. Depending on which forms, strong or weak, of the governing equations in Sections 2.1 and 2.2 are selected, different types of variational formulations and finite element models for the shell problem can be constructed, as shown in Table 1. The displacement boundary conditions for $w(x)$ and $\phi(x)$, as well as the stress/force boundary conditions for $N_{13}(x)$ and $M_{11}(x)$ at $x=0$ and/or $x=L$ can be either essential or natural, depending on the formulation considered. The quality of the finite element solution, as is well known, can largely depend on the variational formulation employed, especially when low-order polynomial approximations are used.

Table 1. An overview of variational formulations

\begin{tabular}{|l|c|c|c|c|}
\hline & \multicolumn{4}{|c|}{ Variational formulation } \\
\cline { 2 - 5 } & primal & primal-mixed & dual-mixed & dual \\
\hline \hline Kinematic equations & strong & 1 st weak & 2nd weak & 2nd weak \\
\hline Constitutive equations & strong & strong & strong & strong \\
\hline Equilibrium equations & 2 nd weak & 2nd weak & 1st weak & strong \\
\hline \hline Displacement b.c. & essential & essential & natural & natural \\
\hline Stress/force b.c. & natural & natural & essential & essential \\
\hline
\end{tabular}

Note that there exist two other primal-dual mixed formulations for the shell problem that are not included in Table 1 . They can be obtained by mixing the weak forms of the governing equations in such a way that one of the kinematic equations is considered in its first weak form, the other in its second weak form, and the corresponding equilibrium equations are taken into account in their second and first weak forms, respectively. Out of the two possibilities, a consistent primal-dual mixed formulation and the related shell finite element are presented in Section 3.4 . Note also that additional mixed variational formulations and finite element models can be constructed by taking into account the constitutive equations in their weak forms.

\section{Finite element Formulations}

In this section, the derivation of the stiffness matrices and the load vectors of one shell element denoted by $e$ will be summarized for displacement-based, primal-mixed, dual-mixed and a consistent primal-dual mixed variational formulations, employing Bubnov-Galerkin-type approximations of the lowest possible order.

The mapping between the master element $\hat{e}:=\{\xi \mid-1 \leq \xi \leq 1\}$ and the actual element $e:=\left\{x \mid x_{a} \leq x \leq x_{b}\right\}$ with nodal coordinates $x_{a}<x_{b}$ is given by

$$
x=x(\xi)=x_{a} \mathcal{N}_{1}(\xi)+x_{b} \mathcal{N}_{2}(\xi),
$$

where

$$
\mathcal{N}_{1}(\xi)=\frac{1}{2}(1-\xi), \quad \mathcal{N}_{2}(\xi)=\frac{1}{2}(1+\xi)
$$


are the standard linear interpolation functions. The Jacobian of the mapping (3.1) is $J=h / 2$ with element length $h=x_{b}-x_{a}$. All the symbolic computations presented in this section have been performed using the computer algebra system Maple $^{T M}$.

3.1. Displacement-based (primal) formulation. This is the simplest and most known formulation, found in many textbooks and papers and reviewed briefly here for notational reasons. The axial membrane force $N_{11}(x)$ is assumed to be known, according to (2.24), and the membrane force $N_{22}(x)$ will be computed from the inverse stress-strain relation 2.20 :

$$
N_{22}(x)=\frac{E d}{R} w(x)+\nu N_{11}(x),
$$

where the kinematic equation $(2.9)$ has also been used. Inserting $(3.3)$, as well as 2.16)-2.17) in 2.33)-2.34 and taking into account 2.7)-2.8, the second weak forms of the equilibrium equations for the shell element $e$ can be written as

$$
\begin{gathered}
\int_{x_{a}}^{x_{b}}\left[-k_{\mathrm{s}} G d\left(w^{e}{ }_{x}+\phi^{e}\right) \delta w^{e}{ }_{x}-\frac{E d}{R^{2}} w^{e} \delta w^{e}+\hat{p}_{3}^{e} \delta w^{e}\right] \mathrm{d} x+\left[N_{13} \delta w\right]_{x_{a}}^{x_{b}}=0 \\
\int_{x_{a}}^{x_{b}}\left[-E_{1} I_{1}\left(\phi^{e},_{x} \delta \phi^{e}{ }_{x}\right)-k_{\mathrm{s}} G d\left(w^{e}{ }_{x}+\phi^{e}\right) \delta \phi^{e}\right] \mathrm{d} x+\left[M_{11} \delta \phi\right]_{x_{a}}^{x_{b}}=0,
\end{gathered}
$$

where $\hat{p}_{3}^{e}=p_{3}^{e}-\nu N_{11}^{e} / R$. Employing linear approximation for both $w(x)$ and $\phi(x)$ on element $e$, we can write:

$$
\begin{aligned}
w^{e}(\xi) & =w_{a} \mathcal{N}_{1}(\xi)+w_{b} \mathcal{N}_{2}(\xi), \\
\phi^{e}(\xi) & =\phi_{a} \mathcal{N}_{1}(\xi)+\phi_{b} \mathcal{N}_{2}(\xi),
\end{aligned}
$$

where $w_{i}=w\left(x_{i}\right)$ and $\phi_{i}=\phi\left(x_{i}\right), i=a, b$ are the nodal displacements and rotations. This is the lowest possible order (though inconsistent) $C^{0}$-continuous approximation for $w(x)$ and $\phi(x)$. Introducing the matrix of nodal displacements

$$
[\mathrm{u}]^{\mathrm{T}}=\left[\begin{array}{llll}
w_{a} & w_{b} & \phi_{a} & \phi_{b}
\end{array}\right]
$$

and approximating the weighting functions $\delta w^{e}(x)$ and $\delta \phi^{e}(x)$ by piece-wise linear functions as well (Bubnov-Galerkin method), the principle of virtual work with variational equations (3.4)-3.5 leads to the matrix equation

$$
\left[\mathrm{K}^{\mathrm{ST}}\right][\mathrm{u}]=\left[\mathrm{F}^{\mathrm{ST}}\right]
$$

where

$$
\begin{aligned}
& {\left[\mathrm{K}^{\mathrm{ST}}\right]=\left[\mathrm{K}_{\mathrm{m}}^{\mathrm{ST}}\right]+\left[\mathrm{K}_{\mathrm{b}}^{\mathrm{ST}}\right]+\left[\mathrm{K}_{\mathrm{s}}^{\mathrm{ST}}\right]=\frac{E d}{h} \frac{h^{2}}{R^{2}}\left[\begin{array}{cccc}
1 / 3 & 1 / 6 & 0 & 0 \\
1 / 6 & 1 / 3 & 0 & 0 \\
0 & 0 & 0 & 0 \\
0 & 0 & 0 & 0
\end{array}\right]} \\
& +\frac{E_{1} I_{1}}{h}\left[\begin{array}{rrrr}
0 & 0 & 0 & 0 \\
0 & 0 & 0 & 0 \\
0 & 0 & 1 & -1 \\
0 & 0 & -1 & 1
\end{array}\right]+\frac{k_{\mathrm{s}} G d}{h}\left[\begin{array}{rrrc}
1 & -1 & -h / 2 & -h / 2 \\
-1 & 1 & h / 2 & h / 2 \\
-h / 2 & h / 2 & h^{2} / 3 & h^{2} / 6 \\
-h / 2 & h / 2 & h^{2} / 6 & h^{2} / 3
\end{array}\right]
\end{aligned}
$$


is the standard stiffness matrix of the shell element (indicated by the letters ST in the superscript) and

$$
\left[\mathrm{F}^{\mathrm{ST}}\right]=\left[\begin{array}{c}
F_{1} \\
F_{2} \\
F_{3} \\
F_{4}
\end{array}\right], \quad F_{j}=\frac{h}{2} \int_{-1}^{+1} \hat{p}_{3}^{e} \mathcal{N}_{j}(\xi) \mathrm{d} \xi+\widetilde{Q}_{j},
$$

is its load vector with $\widetilde{Q}_{j}$ and $\widetilde{M}_{j}, j=1,2$ representing known external loads (edge forces and moments) at the nodes. For the sake of subsequent comparisons, the stiffness matrix in 3.10 is given as the sum of three matrices: $\left[\mathrm{K}_{\mathrm{m}}^{\mathrm{ST}}\right],\left[\mathrm{K}_{\mathrm{b}}^{\mathrm{ST}}\right]$ and $\left[\mathrm{K}_{\mathrm{s}}^{\mathrm{ST}}\right]$ are called, respectively, the membrane, bending and shear part of the stiffness matrix of the shell element.

3.2. Primal-mixed formulation. In the primal-mixed formulation, the equilibrium equations are considered in their second weak forms (2.33)-(2.34), just like in the pure displacement-based formulation: the kinematic equations are, however, taken into account in their first weak forms 2.26$)-(2.27)$ and 2.30 . This allows independent approximations for the stress variables $N_{22}(x), N_{13}(x)$ and $M_{11}(x)$. The membrane force $N_{11}(x)$ is assumed to be known from 2.24). Taking into account the constitutive relations (2.16)-(2.17) and (2.20), the corresponding variational equations for element $e$ read:

$$
\begin{gathered}
\int_{x_{a}}^{x_{b}}\left(\frac{1}{k_{\mathrm{s}} G d} N_{13}^{e}-w^{e}{ }_{x}-\phi^{e}\right) \delta N_{13}^{e} \mathrm{~d} x=0 \\
\int_{x_{a}}^{x_{b}}\left(\frac{1}{E_{1} I_{1}} M_{11}^{e}-\phi^{e}{ }_{x}\right) \delta M_{11}^{e} \mathrm{~d} x=0 \\
\int_{x_{a}}^{x_{b}}\left[\frac{1}{E d}\left(N_{22}^{e}-\nu N_{11}^{e}\right)-\frac{w^{e}}{R}\right] \delta N_{22}^{e} \mathrm{~d} x=0 \\
\int_{x_{a}}^{x_{b}}\left[-N_{13}^{e} \delta w^{e}{ }_{x}-\frac{1}{R} N_{22}^{e} \delta w^{e}+p_{3}^{e} \delta w^{e}\right] \mathrm{d} x+\left[N_{13} \delta w\right]_{x_{a}}^{x_{b}}=0, \\
\int_{x_{a}}^{x_{b}}\left(-M_{11}^{e} \delta \phi^{e},_{x}-N_{13}^{e} \delta \phi^{e}\right) \mathrm{d} x+\left[M_{11} \delta \phi\right]_{x_{a}}^{x_{b}}=0 .
\end{gathered}
$$

As the displacement boundary conditions are still essential, the primal-mixed formulation requires $C^{0}$ continuous approximation for the displacement variables $w(x)$ and $\phi(x)$, and their lowest possible order approximation is linear. The stress boundary conditions are still natural, there is no continuity requirement for the variables $N_{13}(x)$ and $M_{11}(x)$, their lowest possible order approximation is constant. The membrane force $N_{22}(x)$ is considered now as an independent variable and, as no boundary condition exists for it, its lowest order approximation is constant:

$$
\begin{array}{lll}
w^{e}(\xi)=w_{a} \mathcal{N}_{1}(\xi)+w_{b} \mathcal{N}_{2}(\xi), & N_{22}^{e}(\xi)=N_{0}, & \\
\phi^{e}(\xi)=\phi_{a} \mathcal{N}_{1}(\xi)+\phi_{b} \mathcal{N}_{2}(\xi), & N_{13}^{e}(\xi)=Q_{0}, & M_{11}^{e}(\xi)=M_{0}
\end{array}
$$


Introducing the matrices of nodal displacements $w_{i}=w\left(x_{i}\right)$ and $\phi_{i}=\phi\left(x_{i}\right), i=a, b$ and element stresses $N_{0}, Q_{0}, M_{0}$, as

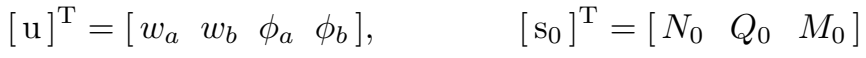

and applying the Bubnov-Galerkin method, i.e., $\delta w^{e}(x)$ and $\delta \phi^{e}(x)$ are approximated by linear functions, while $\delta N_{22}^{e}(x)=\delta N_{0}, \delta N_{13}^{e}(x)=\delta Q_{0}$ and $\delta M_{11}^{e}(x)=\delta M_{0}$ are arbitrary constants, the stress parameters in $\left[s_{0}\right]$ can be eliminated at element level and variational equations 3.12 - 3.16 lead to the matrix equation

$$
\left[\mathrm{K}^{\mathrm{PM}}\right][\mathrm{u}]=\left[\mathrm{F}^{\mathrm{PM}}\right] \text {, }
$$

where

$$
\begin{aligned}
& {\left[\mathrm{K}^{\mathrm{PM}}\right]=\left[\mathrm{K}_{\mathrm{m}}^{\mathrm{PM}}\right]+\left[\mathrm{K}_{\mathrm{b}}^{\mathrm{PM}}\right]+\left[\mathrm{K}_{\mathrm{s}}^{\mathrm{PM}}\right]=\frac{E d}{h} \frac{h^{2}}{R^{2}}\left[\begin{array}{cccc}
1 / 4 & 1 / 4 & 0 & 0 \\
1 / 4 & 1 / 4 & 0 & 0 \\
0 & 0 & 0 & 0 \\
0 & 0 & 0 & 0
\end{array}\right]} \\
& +\frac{E_{1} I_{1}}{h}\left[\begin{array}{rrrr}
0 & 0 & 0 & 0 \\
0 & 0 & 0 & 0 \\
0 & 0 & 1 & -1 \\
0 & 0 & -1 & 1
\end{array}\right]+\frac{k_{\mathrm{s}} G d}{h}\left[\begin{array}{rrrr}
1 & -1 & -h / 2 & -h / 2 \\
-1 & 1 & h / 2 & h / 2 \\
-h / 2 & h / 2 & h^{2} / 4 & h^{2} / 4 \\
-h / 2 & h / 2 & h^{2} / 4 & h^{2} / 4
\end{array}\right]
\end{aligned}
$$

is the stiffness matrix of the primal-mixed element (indicated by the letters PM in the superscript) and

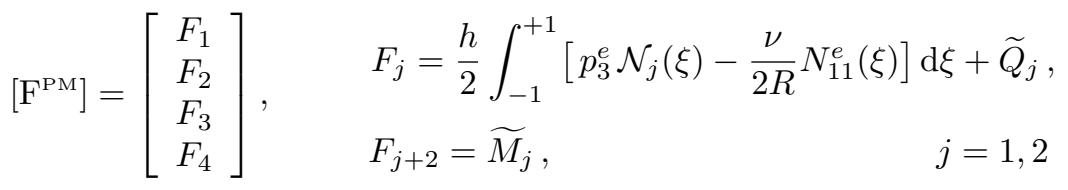

is its load vector with external nodal forces and moments $\widetilde{Q}_{j}$ and $\widetilde{M}_{j}, j=1,2$.

Remark. If $N_{22}(x)$ is not considered as an independently approximated variable but computed from (3.3), then variational equation (3.14) is identically satisfied and the membrane part of the primal-mixed stiffness matrix will be equal to that of the standard displacement-based element, and the equality of the load vectors holds true as well, i.e.,

$$
\left[\mathrm{K}_{\mathrm{m}}^{\mathrm{PM}}\right]=\left[\mathrm{K}_{\mathrm{m}}^{\mathrm{ST}}\right] \quad \text { and } \quad\left[\mathrm{F}^{\mathrm{PM}}\right]=\left[\mathrm{F}^{\mathrm{ST}}\right] .
$$

The shear part of the primal-mixed stiffness matrix does not change, i.e., $\left[\mathrm{K}_{\mathrm{s}}^{\mathrm{PM}}\right]$ is computed according to (3.21) and, thus, it always differs from that of the standard element, $\left[\mathrm{K}_{\mathrm{s}}^{\mathrm{ST}}\right]$.

3.3. Dual-mixed formulation. In the dual-mixed formulation, the kinematic equations are taken into account in their second weak forms $(2.28)-(2.29)$ and the equilibrium equations are considered in their first weak forms (2.31)-(2.32). The axial 
membrane force $N_{11}(x)$ is assumed to be known, according to 2.24), and the membrane force $N_{22}(x)$ will be computed from $(3.3)$. Taking into account the constitutive equations (2.16)-2.17), the corresponding variational equations for element $e$ read:

$$
\begin{gathered}
\int_{x_{a}}^{x_{b}}\left(\frac{1}{k_{\mathrm{s}} G d} N_{13}^{e} \delta N_{13}^{e}+w^{e} \delta N_{13, x}^{e}-\phi^{e} \delta N_{13}^{e}\right) \mathrm{d} x-\left[w \delta N_{13}\right]_{x_{a}}^{x_{b}}=0 \\
\int_{x_{a}}^{x_{b}}\left(\frac{1}{E_{1} I_{1}} M_{11}^{e} \delta M_{11}^{e}+\phi^{e} \delta M_{11, x}^{e}\right) \mathrm{d} x-\left[\phi \delta M_{11}\right]_{x_{a}}^{x_{b}}=0 \\
\int_{x_{a}}^{x_{b}}\left(N_{13, x}^{e}-\frac{E d}{R^{2}} w^{e}+\hat{p}_{3}^{e}\right) \delta w^{e} \mathrm{~d} x=0 \\
\int_{x_{a}}^{x_{b}}\left(M_{11, x}^{e}-N_{13}^{e}\right) \delta \phi^{e} \mathrm{~d} x=0
\end{gathered}
$$

As the displacement boundary conditions are natural in this case, the displacement variables $w(x)$ and $\phi(x)$ can be approximated discontinuously, and their lowest-order approximation is constant. The stress boundary conditions are now essential and $C^{0}$-continuous approximation is required for the stress variables $N_{13}(x)$ and $M_{11}(x)$, i.e., their lowest possible order approximation is linear:

$$
\begin{array}{ll}
w^{e}(\xi)=w_{0}, & N_{13}^{e}(\xi)=Q_{a} \mathcal{N}_{1}(\xi)+Q_{b} \mathcal{N}_{2}(\xi), \\
\phi^{e}(\xi)=\phi_{0}, & M_{11}^{e}(\xi)=M_{a} \mathcal{N}_{1}(\xi)+M_{b} \mathcal{N}_{2}(\xi) .
\end{array}
$$

The matrices of the unknowns, the element displacements $w_{0}, \phi_{0}$ and the nodal stresses $Q_{i}=N_{13}\left(x_{i}\right), M_{i}=M_{11}\left(x_{i}\right), i=a, b$, appearing in (3.27)-(3.28), are given by

$$
\left[\mathrm{u}_{0}\right]^{\mathrm{T}}=\left[\begin{array}{ll}
w_{0} & \phi_{0}
\end{array}\right], \quad[\mathrm{s}]^{\mathrm{T}}=\left[\begin{array}{llll}
Q_{a} & Q_{b} & M_{a} & M_{b}
\end{array}\right]
$$

The element stiffness matrix of the dual-mixed formulation will be derived applying the $\lambda$-multiplier technique, a method often called hybridization [15, 16]. This technique involves the introduction of additional nodal variables as unknowns that turn out to be the nodal values of the displacement $w(x)$ and rotation $\phi(x)$ of element $e$ :

$$
[\mathrm{u}]^{\mathrm{T}}=\left[\begin{array}{llll}
w_{a} & w_{b} & \phi_{a} & \phi_{b}
\end{array}\right]
$$

where $w_{i}=w\left(x_{i}\right)$ and $\phi_{i}=\phi\left(x_{i}\right), i=a, b$. Note that these nodal displacements and rotations are, at least at approximation level, independent from $w_{0}$ and $\phi_{0}$, which are approximations for $w^{e}(x)$ and $\phi^{e}(x)$ in the element domain. In view of (3.29) and 3.30 , the last terms on the left-hand side of 3.23)-3.24 can be written as

$$
\begin{aligned}
-\left[w \delta N_{13}\right]_{x_{a}}^{x_{b}} & =w_{a} \delta Q_{a}-w_{b} \delta Q_{b}, \\
-\left[\phi \delta M_{11}\right]_{x_{a}}^{x_{b}} & =\phi_{a} \delta M_{a}-\phi_{b} \delta M_{b},
\end{aligned}
$$

and, in order to ensure the continuity of $N_{13}(x)$ and $M_{11}(x)$ at the nodes, variational equations (3.23)-(3.26) should be supplemented by equations

$$
\begin{aligned}
\delta w_{a} Q_{a}-\delta w_{b} Q_{b} & =0, \\
\delta \phi_{b} M_{b}-\delta \phi_{b} M_{b} & =0,
\end{aligned}
$$


where $\delta w_{i}=\delta w\left(x_{i}\right)$ and $\delta \phi_{i}=\delta \phi\left(x_{i}\right), i=a, b$ are arbitrary nodal values. Equations (3.33)-(3.34) are parts of the global variational equations that ensure the continuity of the shear force $N_{13}(x)$ and bending moment $M_{11}(x)$ at all the nodes (see Appendix A.

Using the variational equations $(3.23)-(3.26)$ together with $3.31-(3.34)$ and applying the Bubnov-Galerkin method, all the variables of $(3.29)$ can be eliminated at element level and, as $w_{i}$ and $\phi_{i}, i=a, b$ are the nodal displacements and rotations, the stiffness matrix and the load vector of the dual-mixed element can be derived. After performing all the (symbolic) computations, the following matrix equation is obtained:

$$
\left[\mathrm{K}^{\mathrm{DM}}\right][\mathrm{u}]=\left[\mathrm{F}^{\mathrm{DM}}\right]
$$

where

$$
\begin{aligned}
& {\left[\mathrm{K}^{\mathrm{DM}}\right]=\left[\mathrm{K}_{\mathrm{m}}^{\mathrm{DM}}\right]+\left[\mathrm{K}_{\mathrm{b}}^{\mathrm{DM}}\right]+\left[\mathrm{K}_{\mathrm{s}}^{\mathrm{DM}}\right]=\frac{E d}{h} \frac{h^{2}}{R^{2}}\left[\begin{array}{cccc}
1 / 4 & 1 / 4 & 0 & 0 \\
1 / 4 & 1 / 4 & 0 & 0 \\
0 & 0 & 0 & 0 \\
0 & 0 & 0 & 0
\end{array}\right] \frac{1}{C_{\mathrm{m}}}} \\
& +\frac{E_{1} I_{1}}{h}\left[\begin{array}{rrrr}
0 & 0 & 0 & 0 \\
0 & 0 & 0 & 0 \\
0 & 0 & 1 & -1 \\
0 & 0 & -1 & 1
\end{array}\right]+\frac{k_{\mathrm{s}} G d}{h}\left[\begin{array}{cccc}
1 & -1 & -h / 2 & -h / 2 \\
-1 & 1 & h / 2 & h / 2 \\
-h / 2 & h / 2 & h^{2} / 4 & h^{2} / 4 \\
-h / 2 & h / 2 & h^{2} / 4 & h^{2} / 4
\end{array}\right] \frac{1}{C_{\mathrm{s}}}
\end{aligned}
$$

is the stiffness matrix of the dual-mixed element (indicated by the letters DM in the superscript) and

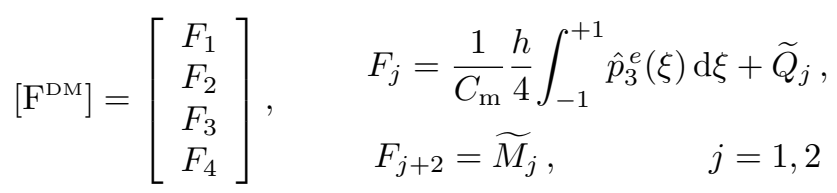

is its load vector with $\widetilde{Q}_{j}$ and $\widetilde{M}_{j}, j=1,2$ representing known external loads at the nodes. The material- and mesh-dependent constants $C_{\mathrm{m}}$ and $C_{\mathrm{s}}$ appearing in 3.36 and (3.37) are given by

$$
\begin{array}{llll}
C_{\mathrm{m}} & =1+\frac{1}{12 k_{\mathrm{s}}} \frac{E}{G} \frac{h^{2}}{R^{2}}, & \text { with } & \lim _{h / R \rightarrow 0} C_{\mathrm{m}}=1, \\
C_{\mathrm{s}} & =1+k_{\mathrm{s}} \frac{G}{E_{1}} \frac{h^{2}}{d^{2}}, & \text { with } & \lim _{h / d \rightarrow 0} C_{\mathrm{s}}=1 .
\end{array}
$$

The role of these constants is discussed in Section 3.5. Note that for isotropic materials $C_{\mathrm{m}}$ and $C_{\mathrm{s}}$ depend on the Poisson ratio only, as $E / G=2(1+\nu)$ and $G / E_{1}=(1-\nu) / 2$.

Remark. If variational equations $(3.23)-(3.26)$ are supplemented by the weak form of the kinematic equation 2.30 or, equivalently, by $(3.14)$, then $N_{22}(x)$ can be approximated as an independent variable. A dual-mixed formulation of this type with piece-wise constant approximation for $N_{22}(x)$ yields, however, the same stiffness matrix as given by (3.36). This is due to the fact that in the above formulation $N_{22}(x)$ is 
expressed by $w(x)$ using $(3.3)$, and $w(x)$ has been approximated by an element-wise constant function.

3.4. A consistent primal-dual mixed formulation. In view of the strong forms of the kinematic equation (2.7) and the equilibrium equation 2.23) of the shell model, a consistent finite element approximation would require that the polynomial degree of the displacement $w(x)$ be higher by one than that of the rotation $\phi(x)$, and the same applies to the bending moment $M_{11}(x)$ and shear force $N_{13}(x)$. In this sense, none of the three finite element formulations and models considered in Sections 3.1 3.3 is consistent, since both $w(x)$ and $\phi(x)$ are approximated by the same degree of polynomials (by either constant, or linear functions) and this is true for $M_{11}(x)$ and $N_{13}(x)$ as well.

A consistent approximation of the lowest possible order, i.e., constant for $\phi(x)$ and $N_{13}(x)$ and linear for $w(x)$ and $M_{11}(x)$, can easily be derived for the shell model investigated by mixing the weak forms $(2.26)-(2.29)$ and $(2.31)-2.34)$ of the governing equations in such a way that one of the kinematic equations is considered in its first weak form, the other in its second weak form, and the equilibrium equations are taken into account accordingly. The corresponding consistent primal-dual mixed weak formulation, out of the two possible formulations of this type, is described by the following variational equations:

$$
\begin{aligned}
\int_{x_{a}}^{x_{b}}\left(\frac{1}{k_{\mathrm{s}} G d} N_{13}^{e}-w^{e}{ }_{x}-\phi^{e}\right) \delta N_{13}^{e} \mathrm{~d} x & =0, \\
\int_{x_{a}}^{x_{b}}\left(\frac{1}{E_{1} I_{1}} M_{11}^{e} \delta M_{11}^{e}+\phi^{e} \delta M_{11, x}^{e}\right) \mathrm{d} x-\left[\phi \delta M_{11}\right]_{x_{a}}^{x_{b}} & =0, \\
\int_{x_{a}}^{x_{b}}\left[-N_{13}^{e} \delta w^{e}{ }_{x}-\frac{E d}{R^{2}} w^{e} \delta w^{e}+\hat{p}_{3}^{e} \delta w^{e}\right] \mathrm{d} x+\left[N_{13} \delta w\right]_{x_{a}}^{x_{b}} & =0, \\
\int_{x_{a}}^{x_{b}}\left(M_{11, x}^{e}-N_{13}^{e}\right) \delta \phi^{e} \mathrm{~d} x & =0 .
\end{aligned}
$$

These equations can be considered as a special combination of the weak forms of the primal- and dual-mixed formulations of Sections 3.2 and 3.3 . As the displacement boundary condition for $w(x)$ and the stress boundary condition for $M_{11}(x)$ are now essential, $C^{0}$-continuous approximation is required for them. The boundary conditions for $\phi(x)$ and $N_{13}(x)$ are natural, they can be approximated discontinuously. The lowest possible order approximation of the variables is given as follows:

$$
\begin{aligned}
w^{e}[\xi(x)] & =w_{a} \mathcal{N}_{1}(\xi)+w_{b} \mathcal{N}_{2}(\xi), & \phi^{e}(x) & =\phi_{0}, \\
M_{11}^{e}[\xi(x)] & =M_{a} \mathcal{N}_{1}(\xi)+M_{b} \mathcal{N}_{2}(\xi), & N_{13}^{e}(x) & =Q_{0} .
\end{aligned}
$$

This approximation is consistent with the strong form of the kinematic equation 2.7 and the equilibrium equation (2.23), as the polynomial degree of $w(x)$ is higher by one than $\phi(x)$, and the same applies to $M_{11}(x)$ and $N_{13}(x)$.

The element stiffness matrix for the present case can be obtained by introducing the nodal rotations $\phi_{a}$ and $\phi_{b}$ as Lagrangian multipliers to ensure the continuity of 
$M_{11}(x)$ at the nodes. The corresponding variational equations are equivalent to 3.32 and (3.34) of the dual-mixed formulation of Section 3.3 and, therefore, they are not repeated here. After eliminating the element unknowns $\phi_{0}, Q_{0}, M_{a}, M_{b}$, the matrix equation for the nodal variables $[\mathrm{u}]^{\mathrm{T}}=\left[\begin{array}{llll}w_{a} & w_{b} & \phi_{a} & \phi_{b}\end{array}\right]$ is obtained, which can be given briefly as

$$
\left(\left[\mathrm{K}_{\mathrm{m}}^{\mathrm{ST}}\right]+\left[\mathrm{K}_{\mathrm{b}}^{\mathrm{ST}}\right]+\left[\mathrm{K}_{\mathrm{s}}^{\mathrm{DM}}\right]\right)[\mathrm{u}]=\left[\mathrm{F}^{\mathrm{ST}}\right] .
$$

According to the indices in the superscripts, the stiffness matrix of the primal-dual mixed element is obtained as the sum of the membrane and bending parts of the displacement-based (standard) stiffness matrix of (3.10) and the shear part of the dual-mixed stiffness matrix (3.36). The element load vector is equivalent to that of the displacement-based formulation given by (3.11).

Remark. In the above formulation, $N_{22}(x)$ is computed from $w(x)$ according to 3.3 and will be $C^{0}$ continuous and piece-wise linear (provided that $N_{11}(x)$ is at most a linear function). If $N_{22}(x)$ is considered as an independent variable and approximated by piece-wise constant function, just like in the case of the primal-mixed formulation of Section 3.2 , then the membrane and bending parts of the primal-dual mixed stiffness matrix will be equal to those of the primal-mixed element and the same applies to the load vectors, i.e.,

$$
\left(\left[\mathrm{K}_{\mathrm{m}}^{\mathrm{PM}}\right]+\left[\mathrm{K}_{\mathrm{b}}^{\mathrm{PM}}\right]+\left[\mathrm{K}_{\mathrm{s}}^{\mathrm{DM}}\right]\right)[\mathrm{u}]=\left[\mathrm{F}^{\mathrm{PM}}\right] .
$$

Note, however, that neither constant nor linear approximation of the membrane force $N_{22}(x)$ is consistent with the strong form of the corresponding equilibrium equation 2.22 .

\subsection{Relationships between stiffness matrices and load vectors of different} variational formulations and finite elements. In view of the results of Sections 3.1 3.4 the analytical comparison of the stiffness matrices and the load vectors gives the following results.

3.5.1. Comparison of the displacement-based and primal-mixed formulations. On comparing the stiffness matrices in (3.10) and (3.21), it can be seen that no difference exists between the bending parts, i.e., $\left[\mathrm{K}_{\mathrm{b}}^{\mathrm{ST}}\right]=\left[\mathrm{K}_{\mathrm{b}}^{\mathrm{PM}}\right]$. Four components in the membrane, as well as in the shear parts of the stiffness matrices are different; these terms are indicated by frames in 3.21 . The load vectors $(3.11)$ and $\sqrt{3.22}$ ) of the two formulations are equivalent, provided the membrane force $N_{11}^{e}$ is zero.

The difference in the membrane stiffness matrices has no significant effect on the performance of the elements, which is due to the coefficient $h^{2} / R^{2}$ and to the fact, that in practical finite element computations the relation $h \ll R$ applies. The rather small difference in the shear parts results, however, in a dramatic change in the approximation properties of the elements for thin shell problems and, as is well known, the primal-mixed formulation leads to shear locking-free displacement computations, just like in the case of the Timoshenko beam element (see, e.g., 2, 18, and [17]). Note that the primal-mixed element of Section 3.2 can also be obtained by the reduced integration technique applied in the standard displacement formulation. 
3.5.2. Comparisons with respect to the dual-mixed formulation. On comparing the element stiffness matrices in (3.21) and (3.36), it can be seen that no difference exists between their bending parts. The membrane and shear parts of the stiffness matrices of the primal- and dual-mixed formulations are related through the constants $C_{\mathrm{m}}$ and $C_{\mathrm{s}}$, respectively, defined by $(3.38)$ and $(3.39)$. The relationships are given briefly by

$$
\left[\mathrm{K}_{\mathrm{m}}^{\mathrm{DM}}\right]=\frac{1}{C_{\mathrm{m}}}\left[\mathrm{K}_{\mathrm{m}}^{\mathrm{PM}}\right], \quad\left[\mathrm{K}_{\mathrm{b}}^{\mathrm{DM}}\right]=\left[\mathrm{K}_{\mathrm{b}}^{\mathrm{PM}}\right], \quad\left[\mathrm{K}_{\mathrm{s}}^{\mathrm{DM}}\right]=\frac{1}{C_{\mathrm{s}}}\left[\mathrm{K}_{\mathrm{s}}^{\mathrm{PM}}\right] .
$$

The load vectors (3.22) and (3.37) for arbitrary distributed load $p_{3}(x)$ are different and they are equivalent only in those cases when the shell element is subjected to concentrated shear forces and bending moments $\widetilde{Q}_{i}$ and $\widetilde{M}_{i}, i=1,2$ at the nodes.

After some algebraic manipulation it can also be pointed out that the relationship between the sum of the bending and shear parts of the standard (displacement-based) element's stiffness matrix and that of the dual-mixed element can be written as

$$
\left[\mathrm{K}_{\mathrm{b}}^{\mathrm{DM}}\right]+\left[\mathrm{K}_{\mathrm{s}}^{\mathrm{DM}}\right]=\frac{1}{C_{\mathrm{s}}}\left(\left[\mathrm{K}_{\mathrm{b}}^{\mathrm{ST}}\right]+\left[\mathrm{K}_{\mathrm{s}}^{\mathrm{ST}}\right]\right)
$$

The sum of the above element stiffness matrices will be equal only when $C_{\mathrm{s}}=1$, i.e., in the limiting case of $h / d \rightarrow 0$. The above relation indicates that the results of the standard displacement formulation can, theoretically, never be equivalent to those of the dual-mixed formulation and, as is well known, the standard element cannot lead to shear locking-free results for shells, no matter how thin or thick the shell is.

3.5.3. Comparisons with respect to the primal-dual mixed formulation. Taking into account the results of Subsection 3.4 and making use of relations 3.47$)-(3.48)$, the matrix equation 3.46 can be rewritten as

$$
\left\{\left[\mathrm{K}_{\mathrm{m}}^{\mathrm{ST}}\right]+\frac{1}{C_{\mathrm{s}}}\left(\left[\mathrm{K}_{\mathrm{b}}^{\mathrm{ST}}\right]+\left[\mathrm{K}_{\mathrm{s}}^{\mathrm{ST}}\right]\right)\right\}[\mathrm{u}]=\left[\mathrm{F}^{\mathrm{ST}}\right] .
$$

This equation suggests that the solution of the primal-dual mixed element for the nodal displacements $[\mathrm{u}$ ] can be directly computed with the knowledge of the stiffness matrix and load vector of the standard shell element using the mesh-dependent constant $C_{\mathrm{s}}$. The bending moment and shear force computation will, however, become equivalent to that of the primal-dual mixed element only in that case when they are computed from the nodal displacement vector $[u]$ without differentiation, according to equations (B.5) B.6 and (B.9) of Appendix B.

Assuming that the primal-dual mixed formulation is locking-free, equation 3.49 suggests that either the mesh-dependent constant $C_{\mathrm{s}}$, defined by $(3.39)$, or its reciprocal

$$
0<\mathscr{L}_{\mathrm{s}}=\frac{1}{C_{\mathrm{s}}} \leq 1
$$

can be considered as a shear locking indicator for the standard shell element and its displacement solution. The closer $\mathscr{L}_{\mathrm{s}}$ is to zero, the more serious the shear locking is. $\mathscr{L}_{\mathrm{s}}=C_{\mathrm{s}}=1$ would indicate absolutely locking-free behavior; it could be attained, however, only when the length $h$ of the element is zero. The function $\mathscr{L}_{\mathrm{s}}=\mathscr{L}_{\mathrm{s}}(h / d)$, depicted in Figure 2 for $\nu=0.3$ and $k_{\mathrm{s}}=5 / 6$, clearly indicates that only a sufficiently 
refined mesh, when $h / d$ is (much) smaller then unity and the corresponding $\mathscr{L}_{\text {s }}$ value is close to 1 , can lead to acceptable numerical results in the case of the standard element. For example, if the element size $h$ is chosen to be equal to the thickness $d$, the error in the displacements is around $20 \%$, with respect to the primal-dual mixed solution, no matter how thin or thick the shell is. Using (3.39), the required mesh size and element number for a given locking-error tolerance in the displacement computation can a priori be determined.

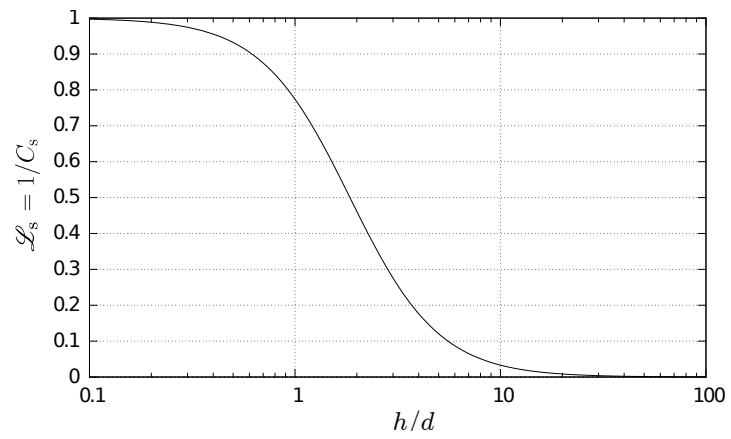

Figure 2. Locking indicator $\mathscr{L}_{\mathrm{s}}=1 / C_{\mathrm{s}}$ versus $h / d$

Remark. The coefficient $C_{\mathrm{m}}$ defined by $(3.38)$ has a very similar structure to $C_{\mathrm{s}}$ in terms of $h / R$ and the function $1 / C_{\mathrm{m}}$ versus $h / R$ is practically the same as that of $1 / C_{\mathrm{s}}$ versus $h / d$ in Figure 2. The relevance of $C_{\mathrm{m}}$ in the finite element computations is, however, not as significant as that of $C_{\mathrm{s}}$, which is primarily due to the fact that relations $h \ll R$ and, thus, $h / R \ll 1$ usually hold true. In addition, the membrane part of the stiffness matrices contains the term $h^{2} / R^{2} \lll 1$ as well.

\section{NumERICAL EXAMPLE AND COMPARISONS}

The analytical comparisons of different variational formulations and finite element matrices, presented in the previous sections, are supplemented here by numerical comparisons through the solution of a simple demonstrative example, a clampedclamped cylindrical shell subjected to uniform load $p_{3}(x)=p_{0}$ (Figure 3). The numerical solutions are computed and compared for shells with thickness to radius ratios $d / R=10^{-2}, 10^{-3}, 10^{-4}$ and $10^{-6}$ employing uniform mesh refinement. The decay length of the cylindrical shell is defined by [19]

$$
\ell=\frac{2 \pi}{\beta} \quad \text { with } \quad \beta=\sqrt[4]{\frac{3\left(1-\nu^{2}\right)}{R^{2} d^{2}}} .
$$

In order to approximate the boundary layer correctly, $L=4 \ell$ is chosen for each shell investigated, and only half the length of the shell is discretized with symmetry conditions prescribed at $x=L / 2=2 \ell$. The radius of the mid-surface is $R=1000 \mathrm{~mm}$, $E=2 \cdot 10^{5} \mathrm{MPa}, \nu=0.3, k_{s}=5 / 6$, and the load value is $p_{0}=1 \mathrm{MPa}$. The finite 
element computations were performed using research codes written in $\mathrm{Maple}^{T M}$ and Octave.

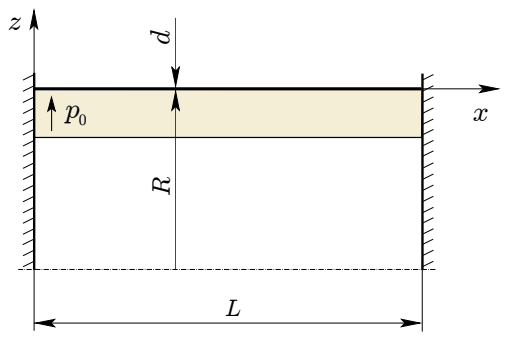

Figure 3. Clamped-clamped shell with uniform load

The decay lengths, as well as the analytical solutions for the central displacement, the maximum bending moment and shear force are listed in Table 2. Scaled values according to the expressions

$$
\bar{w}=w d \quad \bar{M}=\frac{M_{11}}{d}, \quad \bar{Q}=\frac{N_{13}}{\beta d}
$$

are also listed in Table 2, Note that these analytical solutions, based on the Naghdi shell model including shear deformations, are slightly different from that of the Koiter shell model which relies on the Kirchhoff-Love kinematical hypothesis and, thus, excludes shear deformations.

Table 2. Analytical solutions for clamped-clamped shell (Naghdimodel, scaled values)

\begin{tabular}{|c||c|c|c|c|}
\hline$d / R$ & $\ell[\mathrm{mm}]$ & $\left.\bar{w}\right|_{L / 2}$ & $\bar{M}_{\max }$ & $\bar{Q}_{\max }$ \\
\hline \hline $10^{-1}$ & $1.545750 \mathrm{e}+03$ & 4.999982 & $-2.765072 \mathrm{e}+02$ & $5.659172 \mathrm{e}+02$ \\
\hline $10^{-2}$ & $4.888090 \mathrm{e}+02$ & 4.999967 & $-2.997833 \mathrm{e}+02$ & $6.009802 \mathrm{e}+02$ \\
\hline $10^{-3}$ & $1.545750 \mathrm{e}+02$ & 4.999965 & $-3.023283 \mathrm{e}+02$ & $6.047993 \mathrm{e}+02$ \\
\hline $10^{-4}$ & $4.888090 \mathrm{e}+01$ & 4.999965 & $-3.025852 \mathrm{e}+02$ & $6.051847 \mathrm{e}+02$ \\
\hline $10^{-5}$ & $1.545750 \mathrm{e}+01$ & 4.999965 & $-3.026109 \mathrm{e}+02$ & $6.052223 \mathrm{e}+02$ \\
\hline $10^{-6}$ & $4.888090 \mathrm{e}+00$ & 4.999965 & $-3.026135 \mathrm{e}+02$ & $6.052271 \mathrm{e}+02$ \\
\hline
\end{tabular}

The convergence of the central displacement at $L / 2$ is shown in Figure 4 (using logarithmic scale) for the formulations considered in Section 3 . The well-known shear locking phenomenon for the standard displacement-based shell element is clearly seen from the figures. None of the mixed elements are sensitive to the $d / R$ ratio and the asymptotic rate of convergence of the relative error in the displacements is of order two, independently of the mixed formulation employed.

Figure 5 shows the convergence curves of the relative error in the maximum bending moment (at $x=0)$ on a log-log scale. The error amplification due to shear locking 

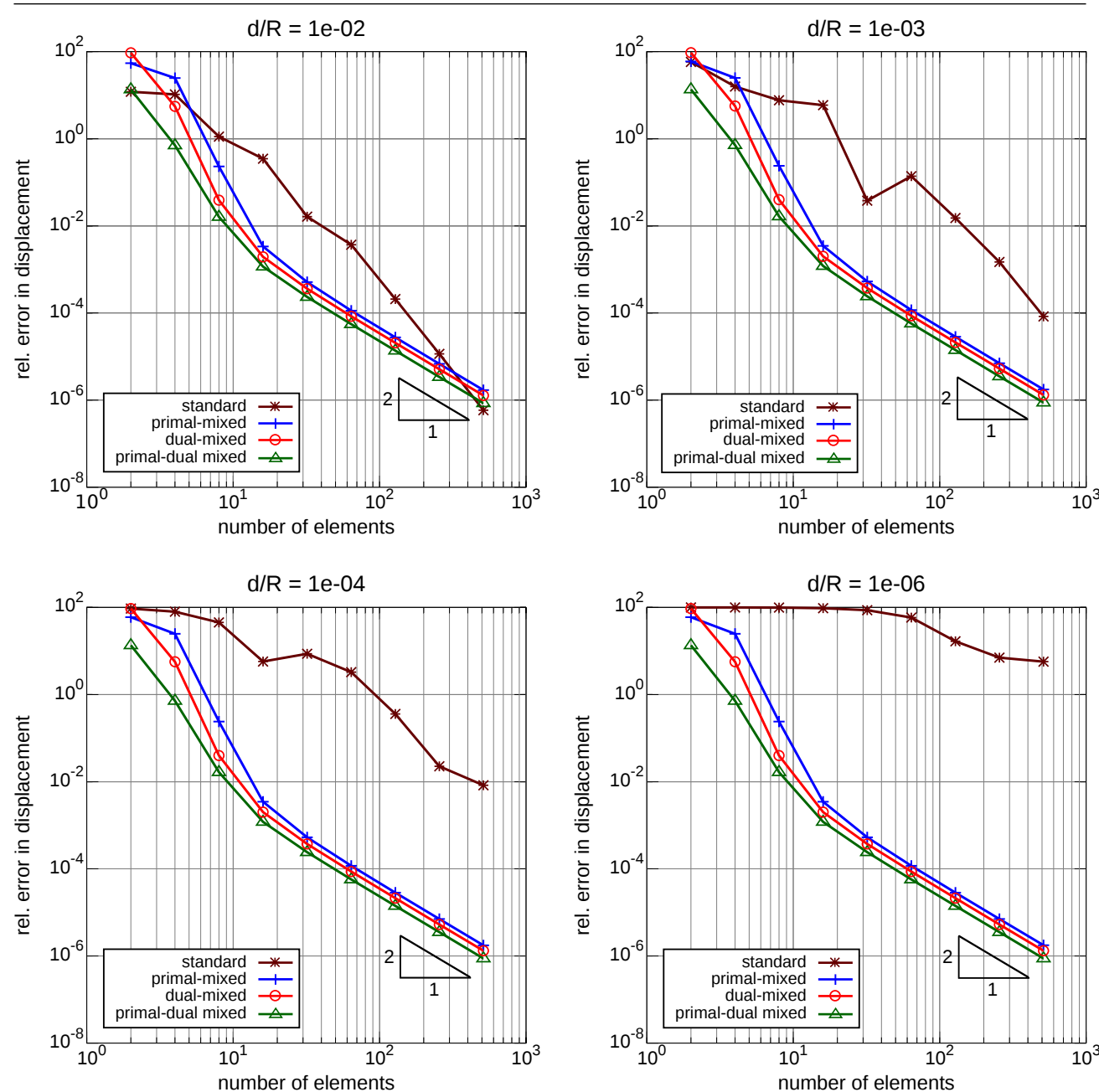

Figure 4. Convergence of the central displacement $w(L / 2)$

of the standard element is apparent again as $d / R$ decreases. The mixed elements are locking-free in the bending moment computations as well; the asymptotic rates of their convergences are, however, significantly different: while the primal-mixed element (equivalent, in this case, with the reduced integration displacement element) gives first-order rates, the dual-mixed and the primal-dual mixed elements give secondorder rates of asymptotic convergence ( $3 \%$ error, for instance, in the bending moment requires about 30 dual-mixed elements and more than 500 primal-mixed elements). This property is clearly the main advantage in the application of the dual-mixed and the primal-dual mixed elements. 

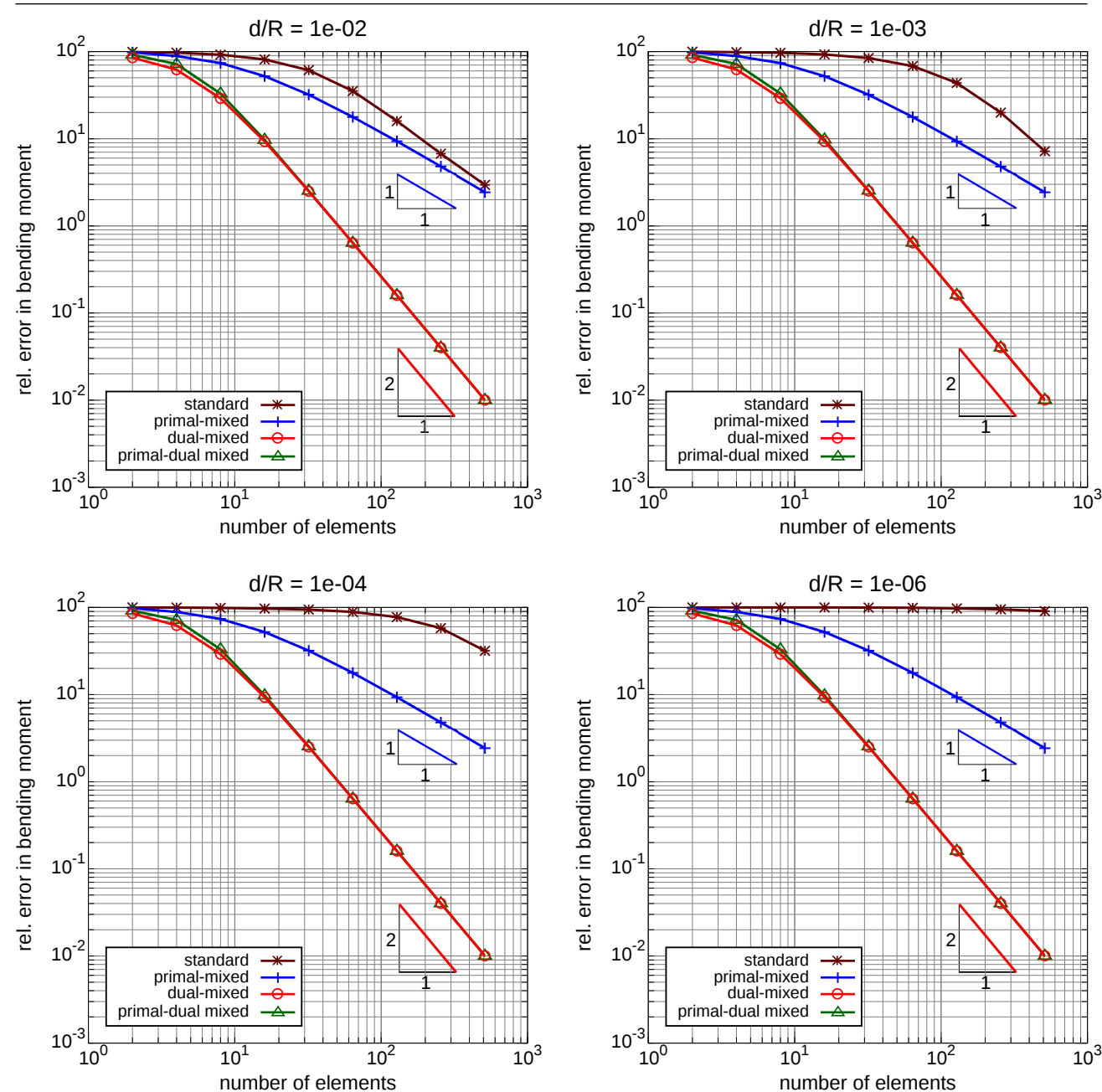

Figure 5. Convergence of the maximum bending moment

The convergence curves of the relative error in the maximum shear force (at $x=0$ ) are depicted in Figure 6. To obtain comparable results for displacement-based and primal-mixed elements, the average nodal values of the first element have been taken as the shear force value at $x=0$. The discretization error due to shear locking of the standard displacement element is the most serious in the shear force computation; the mixed elements are, again, locking-free. Only the dual-mixed element gives secondorder rates of asymptotic convergence for the shear force, whereas the primal-mixed and the primal-dual mixed elements provide first-order rates of convergence only, as indicated in Figure 6. Note that the order of convergence is strongly related to the approximation order of the shear force: in the dual-mixed formulation the 

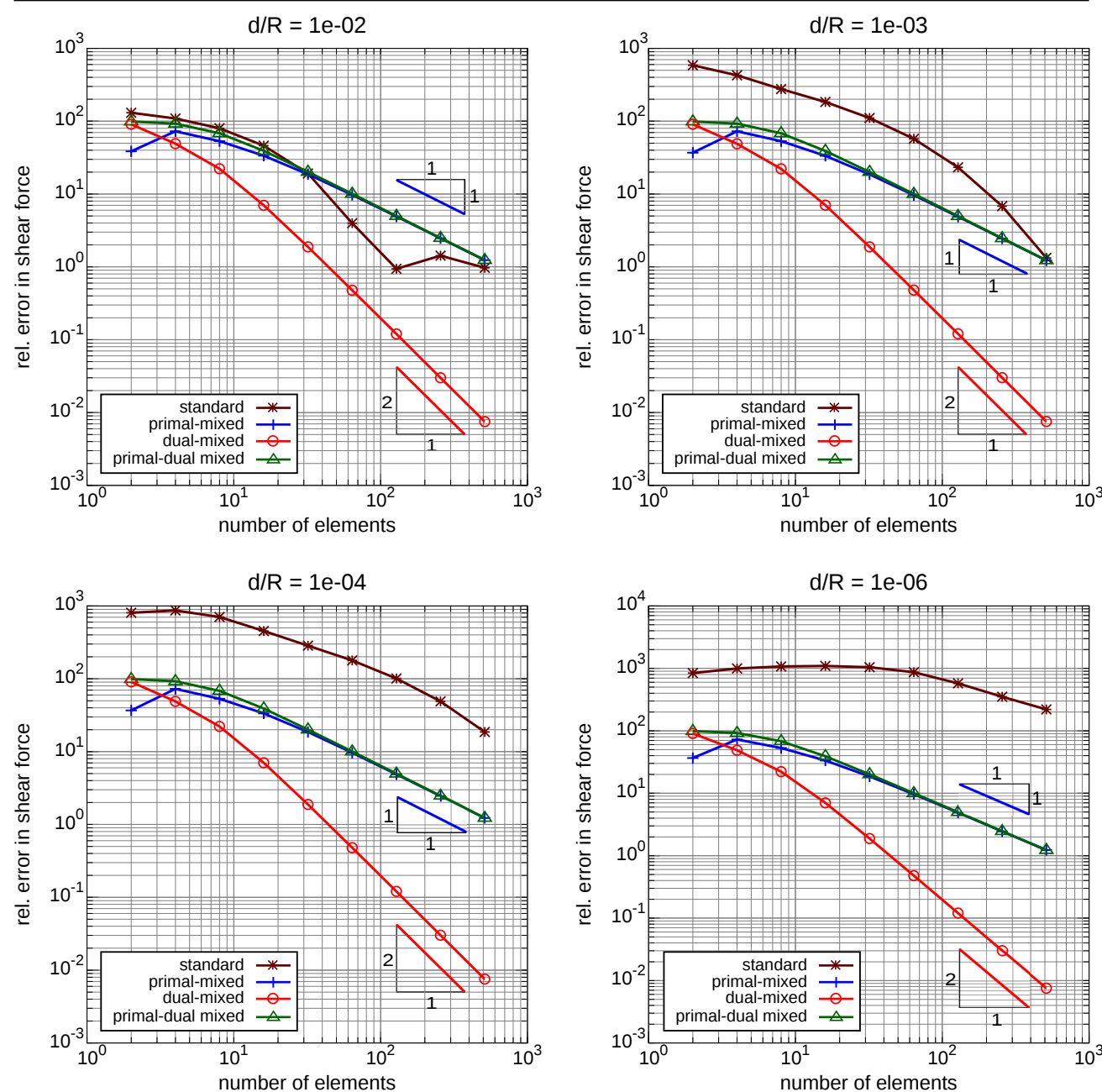

Figure 6. Convergence of the maximum shear force

shear force approximation is element-wise linear and obtained according to (B.7)- B.8 of Appendix B. In the primal-dual-mixed formulation, the shear force is piece-wise constant and obtained according to (B.9).

\section{Concluding Remarks}

Considering the Naghdi shell model for axisymmetric deformations of cylindrical shells, the stiffness matrices and the load vectors of three different mixed finite elements have been derived and compared analytically and explicitly to each other and to those of the standard displacement element, using the lowest possible order 
of polynomial approximations the formulations permit. The shear locking-free behavior of the mixed finite elements has been demonstrated through a simple model problem. The numerical results and comparisons clearly demonstrated that out of the four finite element models investigated in this paper, the dual-mixed element is the only one that gives second-order rates of asymptotic convergence for both the bending moment and shear force computations. This property is considered as the main advantage of the dual-mixed elements over the standard displacement-based and primal-mixed elements.

The analytical investigations of the relationships between the element stiffness matrices resulted in the introduction of two geometry-, material- and mesh-dependent coefficients denoted by $C_{\mathrm{m}}$ and $C_{\mathrm{s}}$ which can easily be computed for each element in the mesh. The effect of $C_{\mathrm{m}}$ is usually insignificant, the coefficient $C_{\mathrm{s}}$, or its reciprocal denoted by $\mathscr{L}_{\mathrm{s}}$ turned out, however, to be a reliable shear locking indicator which can have a significant impact on the behavior of either the displacement-based, or the primal-mixed element. The structure of $C_{\mathrm{s}}$, together with equation 3.49 and the presented numerical results, confirms the well-known fact that locking-free behavior can theoretically never be obtained using the original, unmodified, displacement-based shell element.

The results of the paper provide variational support for the transformability of the standard displacement-based shell element into a shear locking-free dual-mixed one, independently of the thickness and the loading of the shell. The recipe for obtaining shear locking-free solutions for cylindrical shells with second-order rates of convergence in all the variables (displacement, bending moment and shear force) from the knowledge of the stiffness matrix and load vector of the standard displacement formulation and the shear locking indicator $C_{\mathrm{s}}$ of $[3.39]$ is the following:

- compute the standard element stiffness matrix and load vector for each element, according to Subsection 3.1 and equations 3.10)-3.11);

- compute the mesh-dependent constant $C_{\mathrm{s}}$ according to 3.39 for each element of size $h$ in the mesh;

- modify the element stiffness matrices according to (3.48)- 3.49 and, after assembling them, compute the solution for the nodal displacements and rotations;

- in the post-processing phase, compute the element bending moments and shear forces according to $(\mathrm{B} .5)-(\mathrm{B} .6)$ and $(\mathrm{B} .7)-(\mathrm{B} .8)$ of Appendix $\mathrm{B}$.

Similar transformation steps can be applied to the element matrices of the primalmixed formulations, according to equation (3.47), in order to obtain second-order rates of asymptotic convergence in the bending moment and shear force computations.

Acknowledgements. This research was carried out in the framework of the Center of Excellence of Innovative Engineering Design and Technologies at the University of Miskolc and was supported by the New Hungarian Development Plan and jointly financed by the European Union and European Social Fund (TÁMOP-4.2.1.B-10/2/KONV-2010-0001 project). 


\section{REFERENCES}

1. Bishoff, M., Wall, W. A., Bletzinger, K.-U., and Ramm, E.: Models and finite elements for thin walled structures. In The Encyclopedia of Computational Mechanics Vol. II (E. Stein, R. de Borst and T. J. R. Hughes, Eds.). John Wiley \& Sons, New York, 2004. pp. 59-137.

2. Hughes, T. J. R.: The Finite Element Method, Linear Static and Dynamic Finite Element Analysis. Prentice-Hall, Englewood Cliffs, New Jersey, 1987.

3. Bathe, K.-J.: Finite Element Procedures. Prentice-Hall, Upper Saddle River, New Jersey, 1996.

4. Reddy, J. N.: Theory and Analysis of Elastic Plates and Shells. 2nd edn., CRC Press, Boca Raton, 2007.

5. Chapelle, D. and Bathe, K.-J.: The Finite Element Analysis of Shells - Fundamentals. Springer-Verlag, Berlin, 2nd edn., 2011.

6. Arciniega, R. A. and Reddy, J.: Tensor-based finite element formulations for geometrically nonlinear analysis of shells. Computer Methods in Applied Mechanics and Engineering, 196, (2007), 1048-1073.

7. Echter, R., Oesterle, B., and Bischoff, M.: A hierarchic family of isogeometric shell finite elements. Computer Methods in Applied Mechanics and Engineering, 254, (2013), 170-180.

8. Naghdi, P. M.: Foundations of Elastic Shell Theory. In Progress in Solid Mechanics, Vol. IV (I. N. Sneddon and R. Hill, Eds.). North-Holland, Amsterdam, 1963. Pp. 1-90.

9. Kraus, H.: Thin Elastic Shells. John Wiley \& Sons, New York, 1967.

10. Naghdi, P. M.: The Theory of Shells and Plates. In Handbuch der Physik, Band VIa/2 (S. Flügge and C. Truesdell, Eds.). Springer-Verlag, Berlin, 1972. Pp. 425-640.

11. Ciarlet, P. G.: Mathematical Elasticity. Volume III: Theory of Shells. North-Holland, Amsterdam, 2000.

12. PitkÄranta, J.: The problem of membrane locking in finite element analysis of cylindrical shells. Numerische Mathematik, 61, (1992), 523-542.

13. Arnold, D. N. and Brezzi, F.: Locking-free finite element methods for shells. Mathematics of Computation, 66, (1997), 1-14.

14. KlaAs, O., J. Schröder, Stein, E., and Miehe, C.: A regularized dual mixed element for plane elasticity. Implementation and performance of the BDM element. Computer Methods in Applied Mechanics and Engineering, 121, (1995), 201-209.

15. Roberts, J. E. and Thomas, J.-M.: Mixed and Hybrid Methods. In Handbook of Numerical Analysis, Vol. II (P. G. Ciarlet and J. L. Lions, Eds.). North-Holland, Amsterdam, 1991, pp. 523-639.

16. Boffi, D., Brezzi, F., and Fortin, M.: Mixed and Hybrid Finite Element Methods and Applications. Springer-Verlag, New York, 2013.

17. Bertóti, E.: A comparison of primal- and dual-mixed finite element formulations for Timoshenko beams. Engineering with Computers, 31(1), (2015), 99-111.

18. Reddy, J. N.: Energy Principles and Variational Methods in Applied Mechanics. 2nd edn., John Wiley \& Sons, New York, 2002. 
19. Timoshenko, S. P. and Woinowsky-Krieger, S.: Theory of Plates and Shells. McGraw-Hill, New-York, 2nd edn., 2007.

\section{Appendix A.}

In the dual-mixed variational formulation and finite element model presented in Section 3.3 the continuity of the shear force $N_{13}(x)$ and bending moment $M_{11}(x)$ at the element nodes with labels $i=0,1,2, \ldots, n_{e}$ is enforced by the following variational equations:

$$
\begin{aligned}
& \sum_{i=0}^{n_{e}} \delta w_{i} \llbracket Q_{i} \rrbracket=0, \\
& \sum_{i=0}^{n_{e}} \delta \phi_{i} \llbracket M_{i} \rrbracket=0,
\end{aligned}
$$

where $n_{e}$ is the number of the elements, $\delta w_{i}$ and $\delta \phi_{i}$ are arbitrary virtual nodal displacements and rotations, and

$$
\begin{aligned}
\llbracket Q_{i} \rrbracket & =Q_{i}^{i+1}-Q_{i}^{i}, \\
\llbracket M_{i} \rrbracket & =M_{i}^{i+1}-M_{i}^{i}
\end{aligned}
$$

denote the jumps in the shear force and bending moment at node $i$, where $i$ and $i+1$ in the superscript refer to two neighboring elements with common node $i$ in the subscript. Note that A.1 - A.2 represent $2 \times\left(n_{e}+1\right)$ independent equations.

In Section 3.3, the virtual displacements at the nodes of element $e$, with nodal coordinates $x_{a}$ and $x_{b}$, have been denoted by $\delta w_{a} \equiv \delta w_{e-1}, \delta w_{b} \equiv \delta w_{e}$, and the nodal values of the element shear force $Q^{e}(x)$ have been denoted by $Q_{a} \equiv Q_{e-1}^{e} \equiv Q^{e}\left(x_{a}\right)$ and $Q_{b} \equiv Q_{e}^{e} \equiv Q^{e}\left(x_{b}\right)$. Employing this simplified notation, equation A.1 for element $e$ takes the form

$$
\delta w_{a} Q_{a}-\delta w_{b} Q_{b}=0
$$

which proves 3.33 . Applying a similar procedure and notation simplification with respect to equation A.2, variational equation (3.34) is obtained for element $e$.

When concentrated external loads $\widetilde{Q}_{i}$ and $\widetilde{M}_{i}$ are given at node $i$, the jumps in the shear force and bending moment should be equal to these prescribed values. In this case, instead of A.1 - A.2), the modified variational equations

$$
\delta w_{i} \llbracket Q_{i} \rrbracket=\delta w_{i} \widetilde{Q}_{i}, \quad \delta \phi_{i} \llbracket M_{i} \rrbracket=\delta \phi_{i} \widetilde{M}_{i}
$$

hold at node $i$. For completeness it is noted that when displacement boundary conditions are prescribed for the nodal values $w_{i}$ and $\phi_{i}$ at node $i$, then $\delta w_{i}=0$ and $\delta \phi_{i}=0$ in equations A.1 - A.2 and A.6.

\section{Appendix B.}

The dual-mixed formulation of Section 3.3 leads to an equation system of the form

$$
[\mathrm{A}][\mathrm{q}]+[\mathrm{B}][\mathrm{u}]+[\mathrm{f}]=[0],
$$




$$
[\mathrm{B}]^{\mathrm{T}}[\mathrm{q}]+[\mathrm{g}]=[0]
$$

for each element, where, using $\sqrt[3.29]{ },[\mathrm{q}]^{\mathrm{T}}=\left[\left[\mathrm{u}_{0}\right]^{\mathrm{T}}[\mathrm{s}]^{\mathrm{T}}\right]$. An equation system for the nodal variables $[\mathrm{u}]$, defined by 3.30 , can be obtained performing static condensation: expressing the unknowns $[\mathrm{q}]$ from $(\mathrm{B} .1)$ as

$$
[\mathrm{q}]=-[\mathrm{A}]^{-1}([\mathrm{~B}][\mathrm{u}]+[\mathrm{f}])
$$

and substituting B.3 into B.2 leads to equation 3.35 with the following matrix expressions:

$$
\begin{aligned}
& {\left[\mathrm{K}^{\mathrm{DM}}\right][\mathrm{u}]=\left[\mathrm{F}^{\mathrm{DM}}\right], \quad\left[\mathrm{K}^{\mathrm{DM}}\right]=[\mathrm{B}]^{\mathrm{T}}[\mathrm{A}]^{-1}[\mathrm{~B}], } \\
& {\left[\mathrm{F}^{\mathrm{DM}}\right]=[\mathrm{g}]-[\mathrm{B}]^{\mathrm{T}}[\mathrm{A}]^{-1}[\mathrm{f}] . }
\end{aligned}
$$

When the solution for $[\mathrm{u}]$ is known, the bending moment and shear force of the element can be computed using (B.3). For the dual-mixed element of Section 3.3, the nodal bending moments and shear forces are obtained as

$$
\begin{gathered}
M_{a}=\frac{1}{C_{\mathrm{s}}}\left[\frac{k_{\mathrm{s}} G d}{2}\left(w_{a}-w_{b}\right)+\frac{E_{1} I_{1}}{h}\left(\phi_{b}-\phi_{a}\right)-\frac{k_{\mathrm{s}} G h d}{6}\left(2 \phi_{a}+\phi_{b}\right)\right] \\
M_{b}=\frac{1}{C_{\mathrm{s}}}\left[\frac{k_{\mathrm{s}} G d}{2}\left(w_{b}-w_{a}\right)+\frac{E_{1} I_{1}}{h}\left(\phi_{b}-\phi_{a}\right)+\frac{k_{\mathrm{s}} G h d}{6}\left(\phi_{a}+2 \phi_{b}\right)\right] \\
Q_{a}=-\frac{1}{C_{\mathrm{m}}} \frac{E h d}{4 R^{2}}\left(w_{a}+w_{b}\right)+\frac{1}{C_{\mathrm{s}}} k_{\mathrm{s}} G d\left(\frac{w_{b}-w_{a}}{h}+\frac{\phi_{a}+\phi_{b}}{2}\right)+\frac{1}{C_{\mathrm{m}}} \frac{h}{4} \int_{-1}^{+1} \hat{p}_{3}^{e}(\xi) \mathrm{d} \xi \\
Q_{b}=\frac{1}{C_{\mathrm{m}}} \frac{E h d}{4 R^{2}}\left(w_{a}+w_{b}\right)+\frac{1}{C_{\mathrm{s}}} k_{\mathrm{s}} G d\left(\frac{w_{b}-w_{a}}{h}+\frac{\phi_{a}+\phi_{b}}{2}\right)-\frac{1}{C_{\mathrm{m}}} \frac{h}{4} \int_{-1}^{+1} \hat{p}_{3}^{e}(\xi) \mathrm{d} \xi .
\end{gathered}
$$

For the primal-dual mixed element in Section 3.4 the nodal bending moments can be computed according to B.5 $-(B .6)$ and the constant element shear force is

$$
Q_{0}=\frac{1}{C_{\mathrm{s}}} k_{\mathrm{s}} G d\left(\frac{w_{b}-w_{a}}{h}+\frac{\phi_{a}+\phi_{b}}{2}\right) .
$$

The mesh-dependent constants $C_{\mathrm{m}}$ and $C_{\mathrm{s}}$ are given by $3.38-3.39$. 